\title{
Correction to: Controller of Fatigue Testing Machine for Aerospace Thermal Connections Based on Improved NSGA-III Algorithm
}

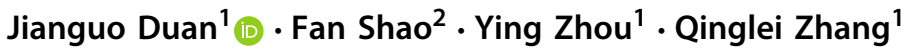

Published online: 7 October 2021

(c) King Fahd University of Petroleum \& Minerals 2021

\section{Correction to:}

\section{Arabian Journal for Science and Engineering} https://doi.org/10.1007/s13369-021-06108-2

In the original publication, the experimental results of section 3.3 and section 3.4 contain errors. The corrected section 3.3 and section 3.4 are as follows:

\subsection{Adaptive crossover operator}

The size of the crossover probability $p_{c}$ has a direct effect on the convergence of the algorithm. If the crossover probability is too large, the speed of new individuals generation would be fast, but the genetic model can be easily destroyed, the crossover probability will be too small, and the search speed would be slow [23]. In order to improve the convergence speed of the NSGA-III algorithm, the crossover operator is improved.

$\mathrm{PC}=\varepsilon-\frac{2 e^{-\frac{n}{N}}}{1+e^{-\frac{n}{N}}} * P_{C}$
Among them, $P_{C}$ indicates a fixed crossover frequency, $\mathrm{n}$ indicates the current number of iterations, $\mathrm{N}$ indicates the maximum number of iterations, $\varepsilon=1.5$.

\subsection{Adaptive mutation operator}

The mutation probability $p_{m}$ affects the search ability of the algorithm. If $p_{m}$ is too large, the individuals with high fitness will be destroyed. The algorithm is similar to random search and the evolution speed is slow. If $p_{m}$ is too small, most individuals will directly enter the next generation, and the search ability will be slow [24]. In order to promote the search ability of the NSGA-III algorithm, the mutation operator has been adaptively promoted.

$\mathrm{PM}=\frac{2 e^{-\frac{n}{N}}}{1+e^{-\frac{n}{N}}} * P_{m}$

Among them, $P_{m}$ indicates a fixed crossover frequency, $\mathrm{n}$ indicates the current number of iterations, $\mathrm{N}$ indicates the maximum number of iterations.

The original article can be found online at https://doi.org/10.1007/s13 369-021-06108-2.

Jianguo Duan

jgduan@shmtu.edu.cn

1 China Institute of FTZ Supply Chain, Shanghai Maritime University, Shanghai 201306, China

2 Institute of Logistics Science and Engineering, Shanghai Maritime University, Shanghai 201306, China 